\title{
Alterações na fertilidade de um solo sob diferentes usos na região de Brasnorte - MT ${ }^{1}$
}

\author{
Cristiane Ramos Vieira²; João Lucas da Silva Araujo ${ }^{3}$; Oscarlina Lúcia dos Santos Weber; José \\ Fernando Scaramuzza ${ }^{5}$
}

Resumo: A conversão da cobertura vegetal natural provoca alterações nas propriedades químicas do solo, de forma a melhorálas ou degradá-las. Este estudo teve como objetivo avaliar as modificações nas propriedades químicas do solo sob diferentes coberturas vegetais, tendo como referência a Floresta Secundária, bioma Cerrado. A pesquisa foi realizada no município de Brasnorte - MT, analisando os sistemas: Floresta Secundária (FS); cultivo de Teca (Tectona grandis) de 19 anos, 16 anos, 13 anos e 8 anos; e Pastagem (PA). Foi utilizado o delineamento em blocos casualizados em esquema fatorial, com as coletas das amostras de solo em três profundidades $(0-5,5-10$ e $10-20 \mathrm{~cm})$ e, posteriormente, feitas as análises químicas. A retirada da vegetação nativa para implantação de cultivo de Teca e de Pastagem alterou as propriedades químicas do solo. O solo sob a Floresta Secundária apresentou os maiores teores de $\mathrm{Ca}^{2+}, \mathrm{K}^{+}$e de $\mathrm{P}$, os maiores valores de soma de bases, de capacidade de troca de cátions e de saturação por bases na camada de $0-5 \mathrm{~cm}$. Os solos sob a Pastagem e sob o cultivo de Teca com 13 anos apresentaram os maiores teores de COT na camada de $0-5 \mathrm{~cm}$, em razão da matéria orgânica formada nos dois casos.

Palavras-chave: Tectona grandis; Floresta Nativa; Fertilidade do Solo; Sistemas de Manejo.

\section{Fertility changes of soil under different uses in region of Brasnorte - MT}

\begin{abstract}
The substitution of natural vegetation causes changes on the chemical properties of soil, in order to improve or degrade them. This study aimed to evaluate the changes in chemical of soil properties under different vegetation cover, with as a reference the Secondary Forest, Cerrado. The study was conducted in the municipality of Brasnorte - MT, considering the following systems: Secondary Forest (FS); Teak cultivation (Tectona grandis) of 19, sixteen years, thirteen years, and eight years; and pasture (PA). The experimental design was randomized blocks in factorial scheme, with soil samples collected at three depths $(0-5,5-10$ and $10-20 \mathrm{~cm})$ and subsequently analyzed chemical and physically. The removal of native vegetation to Teak cultivation and Pasture changed the chemical soil properties. The soil under Secondary Forest showed the highest levels of $\mathrm{Ca}^{2+}, \mathrm{K}^{+}$and $\mathrm{P}$, sum of base values, cation exchange capacity and cation saturation in the $0-5 \mathrm{~cm}$ depth. The soil under the pasture and Teak cultivation with 13 years had the highest TOC in the $0-5 \mathrm{~cm}$ depth, due to the organic matter formed in both cases.
\end{abstract}

Keywords: Tectona grandis; Native Forest; Soil Fertility; Management Systems.

\footnotetext{
${ }^{1}$ Recebido em 04.03.2016 e aceito para publicação como artigo científico em 22.02.2017.

${ }^{2}$ Engenheira Florestal, Dr ${ }^{\mathrm{a}}$. Professora da Universidade de Cuiabá. E-mail: <cris00986@ hotmail.com>.

${ }^{3}$ Engenheiro Florestal. E-mail: <joaolucasaraujo@gmail.com>.

${ }^{4}$ Engenheira Agrônoma, Dra ${ }^{\mathrm{a}}$. Professora Adjunto da Universidade Federal de Mato Grosso. E-mail: <oscsan@uol.com.br>.

${ }^{5}$ Engenheiro Agrônomo, Dr. Professor Adjunto da Universidade Federal de Mato Grosso. E-mail: <jscaramuzza@uol.combr>.
} 


\section{Introdução}

$\mathrm{O}$ aumento da intensidade do uso do solo e a diminuição da cobertura vegetal nativa na região Centro-Norte do Brasil têm levado à perda de recursos naturais e, em especial, à diminuição da fertilidade do solo, por meio da exportação de nutrientes na forma de exploração da madeira nativa e/ou plantada e ainda por conta da substituição da vegetação natural por pastagens e por culturas anuais de menor cobertura vegetal que favorecem a erosão do solo e a decomposição da matéria orgânica (SANTOS e SALCEDO, 2010).

A área em estudo se localiza no município de Brasnorte - MT e se caracteriza por vegetação arbórea nativa do tipo Floresta Ombrófila Aberta que é considerada uma área de transição entre a floresta amazônica e as regiões extraamazônicas. Nessas regiões, a fitomassa e o fitovolume, e por consequência o recobrimento, vão diminuindo gradativamente de densidade. Este fato ocorre em locais com mais de 60 dias secos por ano e em relevos acidentados. A área em questão, frequentemente se caracteriza como a transição entre o cerradão e a floresta ombrófila densa (SILVA, 2007).

O agrossistema de Brasnorte - MT tem na pecuária e na extração de madeira suas principais atividades econômicas. Porém, sabese que a pecuária promove alterações dos recursos naturais, acarretando impactos diretos no solo, no subsolo, na vegetação, na água e na fauna (SILVA et al., 2007). Lima et al. (2011) enfatizam que a conversão de áreas florestais em plantios agrícolas representa uma mudança drástica no ecossistema original, por provocar alterações no conjunto de atributos morfológicos, físicos, químicos e biológicos do solo, rompendo os mecanismos naturais de reciclagem e de proteção do sistema, induzindo a vários fatores de degradação.

Segundo Cardoso et al. (2011), a conversão da vegetação arbórea nativa em pastagem cultivada e o sistema de pastejo contínuo da pastagem nativa no Pantanal Sul-matogrossense promoveram a degradação da qualidade física do solo, através do aumento da densidade e resistência do solo à penetração e diminuição da porosidade total, macroporosidade e condutividade hidráulica.

No aspecto químico, Salgado et al. (2006) avaliaram a fertilidade dos solos em sistema agroflorestal composto por cafés (Coffea arabica), ingazeiros (Inga vera) e grevíleas (Grevillea robusta), sem adubação, sendo: cafeeiro a pleno sol; cafeeiro $\mathrm{x}$ ingazeiro, com árvores distanciadas a $15 \mathrm{~m}$ na linha de plantio e $8 \mathrm{~m}$ na entrelinha; o outro sistema foi o cafeeiro x grevílea, com árvores distanciadas a $10 \mathrm{~m}$ na linha de plantio e $12 \mathrm{~m}$ na entrelinha. E, concluíram que as condições químicas dos solos apresentaram melhores características nos monocultivos de café, o que pode ter ocorrido devido à demanda nutricional das árvores.

Diante dos questionamentos que envolvem o efeito da mudança de cobertura vegetal sobre as propriedades do solo, concebeu-se o presente estudo que objetivou analisar as características químicas do solo sob diferentes coberturas vegetais no município de Brasnorte - MT.

\section{Material e métodos}

O estudo foi realizado na BerTECA Brasil, unidade do grupo Berneck S.A. Painéis e Serrados, localizada na Fazenda Paraná, município de Brasnorte, estado do Mato Grosso - MT. O solo característico da região é do tipo Latossolo Vermelho-Amarelo (LVA). Segundo a Empresa Brasileira de Pesquisa Agropecuária (EMBRAPA, 2006), os latossolos são profundos ou muito profundos, bem drenados, com textura argilosa, muito argilosa ou média. $\mathrm{O}$ clima local é do tipo equatorial quente e úmido, com precipitação anual de $2250 \mathrm{~mm}$ e intensidade máxima em janeiro, fevereiro e março. A temperatura média anual é de $24^{\circ} \mathrm{C}$ (BRASNORTE MT, 2016).

Para o estudo foram escolhidas dez áreas contíguas: cobertura vegetal de Floresta Secundária (FS); Pastagem (PA) com Brachiaria brizantha; cultivo de Teca (Tectona grandis) com oito anos, treze anos, dezesseis anos e dezenove anos; considerando como 
tratamento cada um dos sistemas e os locais de coleta.

O Sistema FS, sob Floresta Ombrófila Aberta, teve sua última interferência antrópica no ano de 1999 com a retirada de espécies de alto valor comercial como: mogno (Swietenia macrophylla), cerejeira (Amburana cearensis) cedro (Cedrela fissilis) e peroba (Aspidosperma polyneuron).

Para a implantação dos sistemas Teca 8, Teca 13, Teca 16 e Teca 19 anos, a floresta primária foi explorada comercialmente e, em seguida, implantou-se o método do "correntão" para a retirada total da vegetação restante, que foi amontoada e queimada. Porém, a área do sistema T16 foi anteriormente utilizada para o cultivo de arroz, em intervalo de três anos consecutivos anteriormente ao plantio. Para todos os sistemas com Teca as mudas foram de origem seminal, do tipo toco raiz nua, produzidas em viveiro próprio, formando área plantada total de 764,33 ha e, cujos tratos culturais, ao longo dos anos, estão descritos na Tabela 1.

Para a obtenção dos dados, para o presente estudo, no mês de maio de 2011, final da estação chuvosa, foram coletadas amostras de solos nas camadas de $0-5,5-10$ e $10-20 \mathrm{~cm}$ de profundidade, a partir da abertura de três mini trincheiras em cada tratamento (sistemas de uso), com no mínimo $30 \mathrm{~cm}$ de profundidade. As características texturais do solo de cada tratamento estão apresentadas na Tabela 2.

As coletas foram realizadas em locais distintos dentro dos sistemas FS e PA. Para o cultivo de Teca com diferentes idades $(8,13,16$ e 19 anos) coletaram-se amostras tanto nas entrelinhas como nas projeções das copas das árvores. Ao todo foram implantados dez tratamentos, sendo eles: Floresta Secundária (FS); Pastagem (PA); T8EL (Teca 8 anos, entre linhas); T13EL; T16EL; T19EL; T8PC (Teca 8 anos, projeção da copa); T13PC; Teca16PC e Teca 19PC.

Para cada tratamento coletaram-se três amostras nas profundidades 0 - 5; 5 - 10 e 10 $20 \mathrm{~cm}$, as quais foram preparadas para serem analisadas química e fisicamente conforme metodologias descritas pela Embrapa (1997). O carbono orgânico total (COT) do solo foi determinado pelo método descrito em Yeomans e Bremner (1988).

Tabela 1 - Tratos silviculturais nos plantios de $T$. grandis.

Table 1 - Silviculture management in $T$. grandis plantations.

Sistema

Teca 8

Teca 13

Teca 19

\section{Tratamentos Silviculturais}

Espaçamento de 3 x $2 \mathrm{~m}$. Sem indicativo do manejo de calagem e/ou adubação. Desbaste no ano de 2009, retirando 50\% do número de árvores, obedecendo a um desbaste baixo do tipo pesado.

Espaçamento de $3 \times 2 \mathrm{~m}$. Sem indicativo do manejo de calagem e/ou adubação. Desbaste no ano de 2002, retirando 30\% do número de árvores, obedecendo a um desbaste baixo do tipo regular. Desbaste no ano de 2007, retirando $40 \%$ do número de árvores, obedecendo a um desbaste sistemático (retirada da sétima linha).

Espaçamento de $3 \times 2 \mathrm{~m}$. Sem indicativo do manejo de calagem e/ou adubação. Desbaste no ano de 1999, retirando $25 \%$ do número de árvores, obedecendo a um desbaste baixo do tipo regular.

Teca 16 Desbaste no ano de 2003, retirando $30 \%$ do número de árvores, obedecendo a um desbaste sistemático (retirada da sétima linha).

Espaçamento de 3 × 2 m. Sem indicativo do manejo de calagem e/ou adubação. Desbaste no ano de 1996, retirando 30\% do número de árvores, obedecendo a um desbaste baixo do tipo regular.

Desbaste no ano de 2002, retirando $40 \%$ do número de árvores, obedecendo a um desbaste sistemático (retirada da sétima linha) em conjunto com um desbaste baixo.

Desbaste no ano de 2008, retirando 55\% do número de árvores, obedecendo a um desbaste baixo. 
Tabela 2 - Textura do solo em função dos tratamentos (sistemas de uso do solo).

Table 2 - Soil texture depending on the treatments (soil use systems).

\begin{tabular}{cccc}
\hline \multirow{2}{*}{ Tratamentos } & Areia & Silte & Argila \\
\cline { 2 - 4 } & & $\left(\mathrm{g} \mathrm{kg}^{-1}\right)$ & 381 \\
Floresta Secundária & 563 & 56 & 693 \\
Pastagem & 256 & 51 & 671 \\
Teca 19 EL & 277 & 52 & 611 \\
Teca 19 PC & 338 & 51 & 580 \\
Teca 16 EL & 396 & 24 & 731 \\
Teca 16 PC & 205 & 64 & 662 \\
Teca 13 EL & 278 & 60 & 642 \\
Teca 13 PC & 311 & 47 & 623 \\
Teca 8 EL & 344 & 33 & 739 \\
Teca 8 PC & 200 & 61 & \\
\hline
\end{tabular}

O delineamento utilizado foi o inteiramente casualizado, contendo três repetições em cada sistema de uso e tipo de amostra (nas entrelinhas ou na projeção da copa), cujos tratamentos consistiram em uma referência (Floresta Secundária) e cinco diferentes sistemas de uso. Utilizando-se, portanto, o esquema fatorial 10x3, com 10 tratamentos (sistemas de cultivo e a Floresta Secundária) com análise de solo em três profundidades.

Os dados foram submetidos ao teste ScottKnott a 5\% de significância quando o teste $\mathrm{F}$ da Análise de Variância (ANOVA) foi significativo a 5\%, utilizando o software SISVAR 4.6 desenvolvido pela Universidade Federal de Lavras - UFLA (FERREIRA, 2003).

\section{Resultados e Discussão}

Houve diferença estatística para os valores de $\mathrm{pH}$ em $\mathrm{CaCl}_{2}$ (Tabela 3) entre tratamentos e em profundidade, ocorrendo interação entre o sistema de cultivo e a profundidade de coleta do solo.

Tabela 3 - pH em função dos tratamentos e das profundidades de amostragem.

Table 3 - pH in function of treatments and sampling depths.

\begin{tabular}{|c|c|c|c|c|}
\hline \multirow{2}{*}{ Tratamento } & \multicolumn{4}{|c|}{$\mathrm{pH}\left(\mathrm{CaCl}_{2}\right)$} \\
\hline & $0-5 \mathrm{~cm}$ & $5-10 \mathrm{~cm}$ & $10-20 \mathrm{~cm}$ & Média \\
\hline Floresta Secundária & $5,69 \mathrm{Aa}$ & $5,39 \mathrm{Bb}$ & $5,22 \mathrm{Cb}$ & 5,4 \\
\hline Pastagem & $4,99 \mathrm{Ca}$ & $4,77 \mathrm{Da}$ & $4,86 \mathrm{Da}$ & 4,9 \\
\hline Teca $19 \mathrm{EL}$ & $5,16 \mathrm{Ba}$ & $4,51 \mathrm{Db}$ & $4,34 \mathrm{~Eb}$ & 4,7 \\
\hline Teca 19 PC & $5,48 \mathrm{Aa}$ & $5,03 \mathrm{Cb}$ & $5,01 \mathrm{Db}$ & 5,2 \\
\hline Teca 16 EL & $5,12 \mathrm{Ba}$ & $4,86 \mathrm{Cb}$ & $4,88 \mathrm{Db}$ & 4,9 \\
\hline Teca 16 PC & $5,62 \mathrm{Ab}$ & $5,68 \mathrm{Ab}$ & $6,06 \mathrm{Aa}$ & 5,8 \\
\hline Teca 13 EL & $5,22 \mathrm{Ba}$ & $4,73 \mathrm{Db}$ & $4,57 \mathrm{~Eb}$ & 4,8 \\
\hline Teca 13 PC & $5,50 \mathrm{Aa}$ & $5,06 \mathrm{Cb}$ & 4,79 Dc & 5,1 \\
\hline Teca 8 EL & $4,87 \mathrm{Ca}$ & $4,62 \mathrm{Db}$ & $4,56 \mathrm{~Eb}$ & 4,7 \\
\hline Teca 8 PC & $5,78 \mathrm{Aa}$ & $5,72 \mathrm{Aa}$ & $5,79 \mathrm{Ba}$ & 5,8 \\
\hline
\end{tabular}

Médias seguidas de mesma letra, maiúscula na coluna e minúscula na linha, não diferem entre si pelo teste Scott-Knott a 5\% de probabilidade.

Os maiores valores de $\mathrm{pH}$ ocorreram na camada superficial de 0 - $5 \mathrm{~cm}$ obtendo-se entre 4,9 e 5,8. Esses valores foram decrescendo com a profundidade, o que pode estar relacionado aos maiores teores de cátions básicos na camada superficial do solo, que eleva o $\mathrm{pH}$ ao aumentar 
a saturação por bases e, ainda, pela maior deposição de material orgânico em superfície, que, com o tempo pode atuar em camadas mais profundas.

No Teca 16 PC, o pH aumentou da profundidade de $5-10 \mathrm{~cm}$ para a de $10-20 \mathrm{~cm}$, enquanto em Teca 8 PC permaneceu semelhante em todas as profundidades. $\mathrm{O}$ aumento nos valores de $\mathrm{pH}$ nos tratamentos Teca $16 \mathrm{PC}$ e Teca $8 \mathrm{PC}$, provavelmente ocorreu devido às cinzas, pois a vegetação anterior foi submetida à queima, considerando que não há registros de adubação na área, apenas de calagem, porém, essa não foi realizada considerando a demanda nutricional da Teca.

Quanto à interpretação do $\mathrm{pH}$, os valores variaram de acidez média (Teca $19 \mathrm{EL}$, Teca 13 EL, Teca 8 EL) a alta (Teca 16 PC e Teca 8 PC), segundo Sousa e Lobato (2004).

Estes resultados são semelhantes aos observados por Carneiro et al. (2009) ao estudarem as propriedades do solo nas coberturas vegetais: cerrado nativo, pastagem, milheto em preparo convencional, nabo forrageiro em plantio direto e sorgo em plantio direto. O que permite indicar que, as alterações realizadas no solo sob floresta nativa secundária para o cultivo de Teca não influenciaram nessa característica química, ou, após alguns anos de cultivo com Teca ocorreu uma melhora nas condições de CTC do solo, o que pode ter culminado no aumento do $\mathrm{pH}$ do solo.

Silva Junior et al. (2012) avaliaram as características químicas do solo considerando uma área de pastagem, um sistema agroflorestal, uma área de mata nativa, uma área de capoeira e uma área com mandioca e verificaram que a redução do $\mathrm{pH}$ em área nativa pode estar relacionada ao fato de que os solos da região já são naturalmente ácidos, o que se agrava ainda mais devido ao processo de degradação da matéria orgânica e da sua rápida mineralização, que acidifica naturalmente o solo.

Em relação aos teores de $\mathrm{Ca}^{2+}$ (Tabela 4), estes decresceram conforme a camada, quando comparados com a Floresta Secundária. Provavelmente, isso se deu porque a calagem nas áreas de Pastagem e de plantio de Teca foi realizada sem considerar as características do solo e sem fazer a manutenção.

Os maiores teores de $\mathrm{Ca}^{2+}$ ocorreram na camada 0 - $5 \mathrm{~cm}$, variando entre 2,13 e 9,27 $\mathrm{cmol}_{\mathrm{c}} \mathrm{dm}^{-3}$, devido aos maiores teores de matéria orgânica acumulada na superfície do solo e à baixa mobilidade do elemento no solo. Mesmo reduzindo com a profundidade em todos os tratamentos, os teores de $\mathrm{Ca}^{2+}$ se enquadraram na faixa adequada segundo Sousa e Lobato (2004) situando-se entre 1,5 e 7,0 $\mathrm{cmol}_{\mathrm{c}} \mathrm{dm}^{-3}$. Porém, observou-se redução dos teores de $\mathrm{Ca}^{2+}$ após a conversão da mata nativa em sistemas de cultivo, indicando que a retirada da vegetação nativa pode influenciar na qualidade química do solo.

Pesquisadores como Sá et al. (2003), analisando floresta secundária, pastagem e café; Lopes et al. (2006) analisando solo sob cultivo de arroz, pastagem e floresta; Melloni et al. (2008) analisando solo sob cultivo de Eucalyptus grandis, Araucaria angustifolia, pastagem e Mata Atlântica e Silva Junior et al. (2012) estudando solo sob área de vegetação mista espontânea tipo capoeira, área de cultivo de mandioca e área de remanescente de Floresta Amazônica, também observaram diferenças nas concentrações de $\mathrm{Ca}^{2+}$ em solo sob diferentes coberturas vegetais.

Apesar de sua baixa mobilidade no solo, observaram-se altos teores de $\mathrm{Ca}^{2+}$ em profundidade na Floresta Secundária, o que pode ter ocorrido em função da contribuição da matéria orgânica na disponibilização de $\mathrm{Ca}^{2+} \mathrm{em}$ maiores profundidades, já que o solo possui textura mais arenosa em relação aos sistemas de cultivo com Teca e a Pastagem. No caso do cultivo de Teca, os teores adequados de $\mathrm{Ca}^{2+}$ pode ter ocorrido, também, devido à decomposição dos resíduos orgânicos que permaneceram no campo após a retirada da mata primitiva no caso da área com Teca 8 PC e, após a retirada do arroz na área com Teca 16 PC. 
Tabela 4 - $\mathrm{Ca}^{2+}$ em função dos tratamentos e das profundidades de amostragem.

Table $4-\mathrm{Ca}^{2+}$ in function of treatments and sampling depths.

\begin{tabular}{|c|c|c|c|c|}
\hline \multirow{2}{*}{ Tratamento } & \multicolumn{4}{|c|}{$\mathrm{Ca}^{2+}\left(\mathrm{cmol}_{\mathrm{c}} \mathrm{dm}^{-3}\right)$} \\
\hline & $0-5 \mathrm{~cm}$ & $5-10 \mathrm{~cm}$ & $10-20 \mathrm{~cm}$ & Média \\
\hline Floresta Secundária & $9,27 \mathrm{Aa}$ & $5,27 \mathrm{Ab}$ & $3,83 \mathrm{Ac}$ & 6,12 \\
\hline Pastagem & $2,13 \mathrm{Ca}$ & $1,70 \mathrm{Ca}$ & $1,37 \mathrm{Ba}$ & 1,73 \\
\hline Teca $19 \mathrm{EL}$ & $4,53 \mathrm{Ba}$ & $2,00 \mathrm{Cb}$ & $1,50 \mathrm{Bb}$ & 2,68 \\
\hline Teca 19 PC & $4,73 \mathrm{Ba}$ & $3,03 \mathrm{Cb}$ & $2,93 \mathrm{Ab}$ & 3,56 \\
\hline Teca 16 EL & $3,17 \mathrm{Ca}$ & $2,17 \mathrm{Ca}$ & $2,13 \mathrm{Ba}$ & 2,49 \\
\hline Teca 16 PC & $3,63 \mathrm{Ba}$ & $3,77 \mathrm{Ba}$ & $3,50 \mathrm{Ba}$ & 3,63 \\
\hline Teca 13 EL & $4,07 \mathrm{Ba}$ & $2,03 \mathrm{Cb}$ & $1,87 \mathrm{Bb}$ & 2,66 \\
\hline Teca 13 PC & $4,77 \mathrm{Ba}$ & $2,67 \mathrm{Cb}$ & $2,17 \mathrm{Bb}$ & 3,20 \\
\hline Teca 8 EL & $2,43 \mathrm{Ca}$ & $1,93 \mathrm{Ca}$ & $1,90 \mathrm{Ba}$ & 2,09 \\
\hline Teca 8 PC & $3,87 \mathrm{Ba}$ & $3,70 \mathrm{Ba}$ & $3,20 \mathrm{Aa}$ & 3,59 \\
\hline
\end{tabular}

Médias seguidas de mesma letra, maiúscula na coluna e minúscula na linha, não diferem entre si pelo teste Scott-Knott a 5\% de probabilidade.

Houve aumento dos teores de $\mathrm{Mg}^{2+}$ com o aumento da profundidade (Tabela 5) na Floresta Secundária, Teca 8 EL e Teca 8 PC. Em geral, os valores de $\mathrm{Mg}^{2+}$ diminuíram da camada superficial para a de $5-10 \mathrm{~cm}$, aumentando em relação à camada de $10-20 \mathrm{~cm}$, o que pode indicar processo de lixiviação (translocação), também descrito por Ramos et al. (2013).

Tabela 5 - $\mathrm{Mg}^{2+}$ em função dos tratamentos e das profundidades de amostragem.

Table $5-\mathrm{Mg}^{2+}$ in function of treatments and sampling depths.

\begin{tabular}{ccccc}
\hline \multirow{2}{*}{ Tratamento } & \multicolumn{4}{c}{$\mathbf{M g}^{\mathbf{2 +}}\left(\mathbf{c m o l}_{\mathbf{~}} \mathbf{~ d m}^{\mathbf{3}}\right)$} \\
\cline { 2 - 5 } & $0-5 \mathrm{~cm}$ & $5-10 \mathrm{~cm}$ & $10-20 \mathrm{~cm}$ & Média \\
\hline Floresta Secundária & $3,13 \mathrm{Aa}$ & $3,37 \mathrm{Aa}$ & $3,97 \mathrm{Aa}$ & 3,49 \\
Pastagem & $1,47 \mathrm{Ba}$ & $0,70 \mathrm{Ca}$ & $0,87 \mathrm{Ca}$ & 1,01 \\
Teca 19 EL & $1,37 \mathrm{Ba}$ & $0,60 \mathrm{Cb}$ & $0,00 \mathrm{Cb}$ & 0,66 \\
Teca 19 PC & $2,13 \mathrm{Aa}$ & $1,67 \mathrm{Ca}$ & $1,73 \mathrm{Ca}$ & 1,84 \\
Teca 16 EL & $1,53 \mathrm{Ba}$ & $1,13 \mathrm{Ca}$ & $1,37 \mathrm{Ca}$ & 1,34 \\
Teca 16 PC & $2,27 \mathrm{Aa}$ & $1,97 \mathrm{Ba}$ & $2,13 \mathrm{Ba}$ & 2,12 \\
Teca 13 EL & $1,87 \mathrm{Ba}$ & $0,73 \mathrm{Ca}$ & $1,27 \mathrm{Ca}$ & 1,29 \\
Teca 13 PC & $2,20 \mathrm{Aa}$ & $2,30 \mathrm{Ba}$ & $2,30 \mathrm{Ba}$ & 2,27 \\
Teca 8 EL & $1,27 \mathrm{Ba}$ & $1,77 \mathrm{Ca}$ & $1,90 \mathrm{Ba}$ & 1,65 \\
Teca 8 PC & $1,77 \mathrm{Ba}$ & $1,33 \mathrm{Ca}$ & $1,83 \mathrm{Ca}$ & 1,64 \\
\hline
\end{tabular}

Médias seguidas de mesma letra, maiúscula na coluna e minúscula na linha, não diferem entre si pelo teste Scott-Knott a 5\% de probabilidade.

No caso da Floresta Secundária, o teor de $\mathrm{Mg}^{2+}$ aumentou com a profundidade, o que pode significar a presença do processo de lixiviação, considerando o regime hídrico médio anual do local, que varia de 2.000 a $3.000 \mathrm{~mm}$, e a cobertura vegetal, em diferentes etapas de degradação devido à diversidade vegetal e à textura do solo mais arenosa que nos demais sistemas. $\mathrm{Ou}$, maiores teores de matéria orgânica em subsuperfície. Portanto, a supressão da vegetação nativa para implantação de cultivo de
Teca e de Pastagem alterou os teores de $\mathrm{Mg}^{2+}$ do solo.

Avaliando-se os teores de $\mathrm{Mg}^{2+}$ verificou-se que estes se enquadraram nas faixas de adequado $\left(0,5-2,0 \mathrm{cmol}_{\mathrm{c}} \mathrm{dm}^{-3}\right)$ e alto (maior que $2,0 \mathrm{cmol}_{\mathrm{c}}$ $\mathrm{dm}^{-3}$ ) segundo Sousa e Lobato (2004). Apesar de o $\mathrm{Mg}^{2+}$ ter maior mobilidade no solo que o $\mathrm{Ca}^{2+}$, os maiores teores foram observados na camada superficial.

O tratamento Floresta Secundária se diferenciou dos demais em relação aos teores de 
$\mathrm{K}^{+}$(Tabela 6), permanecendo semelhante em todas as camadas. Isso auxilia na explicação de que a retirada da mata nativa para implantação de cultivo de Teca e de Pastagem alterou os teores de $\mathrm{K}^{+}$do solo.

Tabela 6 - K em função dos tratamentos e das profundidades de amostragem.

Table 6 - K in function of treatments and sampling depths.

\begin{tabular}{|c|c|c|c|c|}
\hline \multirow{2}{*}{ Tratamento } & \multicolumn{4}{|c|}{$\mathrm{K}\left(\mathrm{mg} \mathrm{dm}^{-3}\right)$} \\
\hline & $0-5 \mathrm{~cm}$ & $5-10 \mathrm{~cm}$ & $10-20 \mathrm{~cm}$ & Média \\
\hline Floresta Secundária & $16,10 \mathrm{Aa}$ & $17,04 \mathrm{Aa}$ & $16,16 \mathrm{Aa}$ & 16,43 \\
\hline Pastagem & $5,96 \mathrm{Ba}$ & $3,13 \mathrm{Cb}$ & $2,31 \mathrm{Bb}$ & 3,80 \\
\hline Teca $19 \mathrm{EL}$ & $6,51 \mathrm{Ba}$ & $4,53 \mathrm{Ca}$ & $2,98 \mathrm{Ba}$ & 4,67 \\
\hline Teca 19 PC & $5,38 \mathrm{Ba}$ & $4,57 \mathrm{Ca}$ & $3,77 \mathrm{Ba}$ & 4,57 \\
\hline Teca 16 EL & $2,40 \mathrm{Ca}$ & $1,28 \mathrm{Ca}$ & $1,16 \mathrm{Ba}$ & 1,61 \\
\hline Teca 16 PC & $5,14 \mathrm{Ba}$ & $3,89 \mathrm{Ca}$ & $3,77 \mathrm{Ba}$ & 4,27 \\
\hline Teca 13 EL & $6,30 \mathrm{Ba}$ & $4,23 \mathrm{Ca}$ & $4,41 \mathrm{Ba}$ & 4,98 \\
\hline Teca 13 PC & $4,99 \mathrm{Bb}$ & $8,25 \mathrm{Ba}$ & $5,29 \mathrm{Bb}$ & 6,18 \\
\hline Teca 8 EL & $2,95 \mathrm{Ca}$ & $1,95 \mathrm{Ca}$ & $1,71 \mathrm{Ba}$ & 2,20 \\
\hline Teca 8 PC & $5,93 \mathrm{Ba}$ & $3,23 \mathrm{Cb}$ & $2,86 \mathrm{Bb}$ & 4,01 \\
\hline
\end{tabular}

Médias seguidas de mesma letra, maiúscula na coluna e minúscula na linha, não diferem entre si pelo teste Scott-Knott a 5\% de probabilidade.

No presente estudo, ao considerar todos os tratamentos, os maiores teores de $\mathrm{K}^{+}$foram observados na camada $0-5 \mathrm{~cm}$. No entanto, foram considerados baixo em todos os tratamentos, de acordo com a interpretação da análise do solo estabelecida por Souza e Lobato (2004). Isso compromete o crescimento e/ou produtividade das culturas, já que o $\mathrm{K}^{+}$é um elemento bastante exigido pelas espécies florestais.

Apesar do decréscimo nos teores de $\mathrm{K}^{+}$com a profundidade, pode-se observar aumento da camada 5 - 10 para a de 10 - $20 \mathrm{~cm}$ em Teca 13 EL e; aumento de 0 - 5 para 5 - 10, diminuindo para $10-20 \mathrm{~cm}$. Provavelmente, devido à presença do processo de lixiviação entre camadas, que geralmente ocorre com os cátions básicos presentes no solo. Sá et al. (2003), analisando solo sob floresta secundária, pastagem e café; Lopes et al. (2006), analisando solo sob área de arroz, de pastagem e de floresta e; Silva Junior et al. (2012), analisando solo sob área de pasto, sistema agroflorestal, mata nativa, área de capoeira e cultivo de mandioca, também observaram teores baixos de $\mathrm{K}^{+}$.

Esses teores baixos para $\mathrm{K}^{+}$podem ter ocorrido em função da mineralogia do solo, da erosão e lixiviação do elemento já que este é móvel no solo, além da absorção pelas plantas e exportação de nutrientes mediante o desbaste, o que comprometeu o potencial produtivo do solo.

Para os teores de P, não se observou diferença entre a Floresta Secundária e os tratamentos Teca 16 EL, Teca 13 PC, Teca 8 EL e Teca 8 PC (Tabela 7) na camada de 0 - $5 \mathrm{~cm}$, verificandose, nesse caso, decréscimo nos teores do elemento conforme a aumento da profundidade. Nos tratamentos PA, Teca 19 PC e Teca 16 EL houve aumento dos teores de $\mathrm{P}$ da camada de 0 5 para a de $5-10 \mathrm{~cm}$, diminuindo para a camada de $10-20 \mathrm{~cm}$.

Os valores $0,00 \mathrm{mg} \mathrm{dm}^{-3}$ de $\mathrm{P}$ na profundidade de $10-20 \mathrm{~cm}$ foram estatisticamente semelhantes aos valores da profundidade de $5-10 \mathrm{~cm}$. O que pode ter ocorrido porque para as maiores profundidades se observaram as maiores variações entre as profundidades estudadas, atingindo CV de $114 \%$ em profundidade e de $112 \%$ entre os tratamentos.

Em geral, os teores de $\mathrm{P}$ foram considerados baixos, segundo Sousa e Lobato (2004). Sá et al. (2003) também observaram teores baixos de P, explicando que, estes estão coerentes com o que são observados nas regiões de clima tropical, assim como foi constatado na área da presente 
pesquisa. Lopes et al. (2006) e Silva Junior et al. (2012) também observaram teores baixos de P em função dos diferentes usos do solo. Isso ocorre porque, em condições naturais de solos de Cerrado, cujo grau de intemperismo está avançado, o solo é rico em coloides inorgânicos capazes de promover a adsorção do $\mathrm{Pe}$, portanto, deixá-lo em uma forma não absorvível pelas plantas.

Tabela 7 - P em função dos tratamentos e das profundidades de amostragem.

Table 7 - P in function of treatments and sampling depths.

\begin{tabular}{ccccc}
\hline \multirow{2}{*}{ Tratamento } & \multicolumn{4}{c}{$\mathbf{P}\left(\mathbf{m g ~ d m}^{-\mathbf{3}}\right)$} \\
\cline { 2 - 5 } & $0-5 \mathrm{~cm}$ & $5-10 \mathrm{~cm}$ & $10-20 \mathrm{~cm}$ & Média \\
\hline Floresta Secundária & $5,54 \mathrm{Aa}$ & $2,05 \mathrm{Ab}$ & $0,00 \mathrm{Ab}$ & 2,53 \\
Pastagem & $1,04 \mathrm{Ba}$ & $1,65 \mathrm{Aa}$ & $0,00 \mathrm{Aa}$ & 0,90 \\
Teca 19 EL & $1,74 \mathrm{Ba}$ & $0,24 \mathrm{Aa}$ & $0,00 \mathrm{Aa}$ & 0,66 \\
Teca 19 PC & $0,72 \mathrm{Ba}$ & $1,48 \mathrm{Aa}$ & $0,64 \mathrm{Aa}$ & 0,95 \\
Teca 16 EL & $3,45 \mathrm{Aa}$ & $5,00 \mathrm{Aa}$ & $4,04 \mathrm{Aa}$ & 4,16 \\
Teca 16 PC & $1,04 \mathrm{Ba}$ & $0,44 \mathrm{Aa}$ & $0,24 \mathrm{Aa}$ & 0,57 \\
Teca 13 EL & $2,25 \mathrm{Ba}$ & $1,00 \mathrm{Aa}$ & $0,00 \mathrm{Aa}$ & 1,08 \\
Teca 13 PC & $3,72 \mathrm{Aa}$ & $1,20 \mathrm{Aa}$ & $0,00 \mathrm{Aa}$ & 1,64 \\
Teca 8 EL & $6,37 \mathrm{Aa}$ & $3,64 \mathrm{Aa}$ & $0,80 \mathrm{Aa}$ & 3,60 \\
Teca 8 PC & $3,55 \mathrm{Aa}$ & $0,84 \mathrm{Aa}$ & $0,00 \mathrm{Aa}$ & 1,46 \\
\hline
\end{tabular}

Médias seguidas de mesma letra, maiúscula na coluna e minúscula na linha, não diferem entre si pelo teste Scott-Knott a 5\% de probabilidade.

A soma de bases (SB) (Tabela 8) foi menor nas camadas 5 - 10 e $10-20 \mathrm{~cm}$ para todos os tratamentos, quando comparado com a Floresta Secundária, que, portanto, apresentou os maiores valores em todas as camadas. Estes resultados foram semelhantes aos observados por Sá et al.
(2003), considerando floresta, pastagem e café. E, estão coerentes, por conta dos teores de $\mathrm{Ca}^{2+}$, $\mathrm{Mg}^{2+}$ e de $\mathrm{K}^{+}$observados em solo sob a cobertura vegetal nativa, indicando que a retirada dessa vegetação reduz os teores de cátions básicos do solo e, consequentemente, a SB.

Tabela 8 - SB em função dos tratamentos e das profundidades de amostragem.

Table 8 - SB in function of treatments and sampling depths.

\begin{tabular}{|c|c|c|c|c|}
\hline \multirow{2}{*}{ Tratamento } & \multicolumn{4}{|c|}{ SB $\left(\right.$ cmolc dm $\left.^{-3}\right)$} \\
\hline & $0-5 \mathrm{~cm}$ & $5-10 \mathrm{~cm}$ & $10-20 \mathrm{~cm}$ & Média \\
\hline Floresta Secundária & $12,44 \mathrm{Aa}$ & $8,68 \mathrm{Ab}$ & $7,84 \mathrm{Ab}$ & 9,65 \\
\hline Pastagem & $3,62 \mathrm{Ca}$ & $2,41 \mathrm{Ca}$ & $2,24 \mathrm{Ca}$ & 2,76 \\
\hline Teca $19 \mathrm{EL}$ & $5,92 \mathrm{Ba}$ & $2,61 \mathrm{Cb}$ & $1,51 \mathrm{Cb}$ & 3,35 \\
\hline Teca 19 PC & $6,88 \mathrm{Ba}$ & $4,71 \mathrm{Bb}$ & $4,68 \mathrm{Bb}$ & 5,42 \\
\hline Teca 16 EL & $4,71 \mathrm{Ca}$ & $3,5 \mathrm{Ca}$ & $3,30 \mathrm{Ca}$ & 3,84 \\
\hline Teca 16 PC & $5,91 \mathrm{Ba}$ & $5,74 \mathrm{Ba}$ & $5,64 \mathrm{Ba}$ & 5,76 \\
\hline Teca 13 EL & $5,95 \mathrm{Ba}$ & $3,31 \mathrm{Cb}$ & $2,62 \mathrm{Cb}$ & 3,96 \\
\hline Teca 13 PC & $6,98 \mathrm{Ba}$ & $4,99 \mathrm{Bb}$ & $4,48 \mathrm{Bb}$ & 5,48 \\
\hline Teca 8 EL & $3,71 \mathrm{Ca}$ & $2,44 \mathrm{Ca}$ & $1,90 \mathrm{Ca}$ & 2,68 \\
\hline Teca 8 PC & $5,65 \mathrm{Ba}$ & $5,04 \mathrm{Ba}$ & $5,04 \mathrm{Ba}$ & 5,24 \\
\hline
\end{tabular}

Médias seguidas de mesma letra, maiúscula na coluna e minúscula na linha, não diferem entre si pelo teste Scott-Knott a $5 \%$ de probabilidade.

Os maiores valores de SB, 3,62 a 12,44 $\mathrm{cmol}_{\mathrm{c}}$. $\mathrm{dm}^{-3}$, ocorreram na camada de $0-5 \mathrm{~cm}$, decrescendo conforme a profundidade. Nesse caso, os tratamentos Teca 16 EL e Teca 8 EL 
apresentaram valores de SB iguais aos de Pastagem. Valores que foram influenciados, provavelmente, pelos teores de $\mathrm{Ca}^{2+}$ e de $\mathrm{Mg}^{2+}$ nesses tratamentos.
A capacidade total de troca de cátions (CTC total) (Tabela 9) também foi reduzida com a retirada da vegetação nativa, o que está coerente com os dados obtidos na análise de SB.

Tabela 9 - CTC total em função dos tratamentos e das profundidades de amostragem.

Table 9 - Total CTC in function of treatments and sampling depths.

\begin{tabular}{|c|c|c|c|c|}
\hline \multirow{2}{*}{ Tratamento } & \multicolumn{4}{|c|}{ CTC total $\left(\mathrm{cmol}_{\mathrm{c}} \mathrm{dm}^{-3}\right)$} \\
\hline & $0-5 \mathrm{~cm}$ & $5-10 \mathrm{~cm}$ & $10-20 \mathrm{~cm}$ & Média \\
\hline Floresta Secundária & $15,63 \mathrm{Aa}$ & $11,79 \mathrm{Ab}$ & $1,95 \mathrm{Db}$ & 6,87 \\
\hline Pastagem & $8,22 \mathrm{Ba}$ & $6,87 \mathrm{Ba}$ & $6,19 \mathrm{Ca}$ & 7,09 \\
\hline Teca $19 \mathrm{EL}$ & $9,81 \mathrm{Ba}$ & $7,10 \mathrm{Bb}$ & $5,71 \mathrm{Cb}$ & 7,54 \\
\hline Teca 19 PC & $10,00 \mathrm{Ba}$ & $8,66 \mathrm{Ba}$ & $8,09 \mathrm{Aa}$ & 8,92 \\
\hline Teca 16 EL & $8,54 \mathrm{Ba}$ & $7,28 \mathrm{Ba}$ & $6,96 \mathrm{Ca}$ & 7,59 \\
\hline Teca 16 PC & $8,77 \mathrm{Ba}$ & $8,26 \mathrm{Ba}$ & 7,91 Ba & 8,31 \\
\hline Teca 13 EL & $9,54 \mathrm{Ba}$ & $7,01 \mathrm{Bb}$ & $6,85 \mathrm{Cb}$ & 7,80 \\
\hline Teca 13 PC & $8,22 \mathrm{Ba}$ & $6,98 \mathrm{Ba}$ & $6,48 \mathrm{Ca}$ & 7,23 \\
\hline Teca 8 EL & $7,25 \mathrm{Ba}$ & $6,13 \mathrm{Ba}$ & $4,83 \mathrm{Cb}$ & 6,07 \\
\hline Teca 8 PC & $9,00 \mathrm{Ba}$ & $8,47 \mathrm{Ba}$ & $8,09 \mathrm{Aa}$ & 8,52 \\
\hline
\end{tabular}

Médias seguidas de mesma letra, maiúscula na coluna e minúscula na linha, não diferem entre si pelo teste Scott-Knott a 5\% de probabilidade.

No caso da CTC total, houve redução significativa em profundidade para Floresta Secundária, Teca 19 EL e Teca 13 EL. Na camada de 10 a $20 \mathrm{~cm}$, os plantios de Teca com 16 e 8 anos apresentaram os maiores valores. Enquanto que, a Floresta Secundária apresentou os menores valores, seguindo a tendência da soma de bases, dada a influência direta dos teores de $\mathrm{Ca}^{2+} \mathrm{e} \mathrm{Mg}^{2+}$.

Em geral, os valores de CTC total se enquadraram nas faixas: baixo $(1,61-4,30$ $\left.\mathrm{cmol}_{\mathrm{c}} \mathrm{dm}^{-3}\right)$, médio $\left(4,31-8,60 \mathrm{cmol}_{\mathrm{c}} \mathrm{dm}^{-3}\right)$, bom $\left(8,61-15,00 \mathrm{cmol}_{\mathrm{c}} \mathrm{dm}^{-3}\right)$ e muito bom (> $\left.15,00 \mathrm{cmol}_{\mathrm{c}} \mathrm{dm}^{-3}\right)$. Sá et al. (2003) observaram resultados semelhantes, atribuindo-os à diversidade de matéria orgânica presente na floresta secundária, em relação à pastagem e à área de café.

Observou-se redução nos valores de saturação por base (V\%) (Tabela 10) com o aumento da profundidade. No caso da Floresta Secundária esses resultados estão relacionados aos teores de $\mathrm{Ca}^{2+}, \mathrm{Mg}^{2+}$ e de $\mathrm{K}^{+}$que elevaram a $\mathrm{SB}$, a CTC e, dessa forma, a V\%. No entanto, em geral, os maiores valores de $\mathrm{V} \%$ foram observados na camada $0-5 \mathrm{~cm}$, assim como verificado por Sá et al. (2003), considerando floresta secundária, pastagem e café.

$\mathrm{O}$ melhor tratamento na camada $0-5 \mathrm{~cm}$ foi o Teca 13 PC apresentando $\mathrm{V} \%=85$, provavelmente devido à serapilheira formada nesse tipo de cultivo, já que a Teca possui material vegetal mais difícil de degradar. Valores de V abaixo de 50\% foram observados na Pastagem, Teca 19 EL, Teca 13 EL e na Teca 8 anos EL, caracterizados como solos distróficos.

Nas camadas de 5 - 10 e de $10-20 \mathrm{~cm}$ os melhores tratamentos foram Floresta Secundária, Teca $16 \mathrm{PC}$ e Teca $13 \mathrm{PC}$, auxiliando a entender o aumento do $\mathrm{pH}$ em profundidade nos mesmos. Porém, em geral, os valores de V\% foram considerados: adequados (36 a $60 \%$ ), alto (61 a $70 \%$ ) e muito alto (maior que $70 \%$ ), segundo Sousa e Lobato (2004). Portanto, os cultivos de Teca se assemelharam à Floresta Secundária, o que significa formar uma camada superficial de solo eutrófica, ou seja, mais fértil (acima de 50\%). 
Tabela 10 - V em função dos tratamentos e das profundidades de amostragem.

Table $10-V$ in function of treatments and sampling depths.

\begin{tabular}{ccccc}
\hline \multirow{2}{*}{ Tratamento } & \multicolumn{4}{c}{$\mathbf{V}(\boldsymbol{\%})$} \\
\cline { 2 - 5 } & $0-5 \mathrm{~cm}$ & $5-10 \mathrm{~cm}$ & $10-20 \mathrm{~cm}$ & Média \\
\hline Floresta Secundária & $73,53 \mathrm{Ba}$ & $69,10 \mathrm{Aa}$ & $67,69 \mathrm{Aa}$ & 70,11 \\
Pastagem & $42,61 \mathrm{Da}$ & $36,13 \mathrm{Ca}$ & $34,82 \mathrm{Da}$ & 37,85 \\
Teca 19 EL & $58,97 \mathrm{Ca}$ & $35,28 \mathrm{Cb}$ & $25,71 \mathrm{Ec}$ & 39,99 \\
Teca 19 PC & $68,17 \mathrm{Ba}$ & $58,23 \mathrm{Bb}$ & $53,84 \mathrm{Bb}$ & 60,08 \\
Teca 16 EL & $54,49 \mathrm{Ca}$ & $48,12 \mathrm{Ba}$ & $47,34 \mathrm{Ca}$ & 49,98 \\
Teca 16 PC & $71,18 \mathrm{Ba}$ & $68,61 \mathrm{Aa}$ & $67,14 \mathrm{Aa}$ & 68,98 \\
Teca 13 EL & $62,15 \mathrm{Ca}$ & $46,69 \mathrm{Bb}$ & $37,44 \mathrm{Db}$ & 48,76 \\
Teca 13 PC & $85,10 \mathrm{Aa}$ & $71,76 \mathrm{Aa}$ & $70,62 \mathrm{Ab}$ & 75,83 \\
Teca 8 EL & $49,82 \mathrm{Da}$ & $39,63 \mathrm{Cb}$ & $38,93 \mathrm{Db}$ & 42,79 \\
Teca 8 PC & $62,68 \mathrm{Ca}$ & $61,97 \mathrm{Ba}$ & $59,54 \mathrm{Ba}$ & 61,40 \\
\hline
\end{tabular}

Médias seguidas de mesma letra, maiúscula na coluna e minúscula na linha, não diferem entre si pelo teste Scott-Knott a 5\% de probabilidade.

A saturação por alumínio $(\mathrm{m} \%)$ apresentada na Tabela 11 aumentou com a profundidade, o que pode ser explicado pela redução no $\mathrm{pH}$ e consequente saturação do $\mathrm{Al}^{3+}$ no complexo de troca do solo, já que são características diretamente relacionadas.

Não se observou diferença, na camada 0 - 5 $\mathrm{cm}$; enquanto que, na camada $5-10 \mathrm{~cm}$ os maiores valores foram observados em Teca 19 EL, Teca 13 EL e Teca 8 EL, e; em Teca 19 EL na camada de $10-20 \mathrm{~cm}$. Isso indica que o solo pode apresentar a necessidade de intervenção com a calagem e adubação de manutenção com o objetivo de aumentar os teores de bases e, consequentemente o $\mathrm{pH}$, para reduzir $\mathrm{m} \%$.

Tabela 11 - m\% em função dos tratamentos e das profundidades de amostragem.

Table $11-\mathrm{m} \%$ in function of treatments and sampling depths.

\begin{tabular}{|c|c|c|c|c|}
\hline \multirow{2}{*}{ Tratamento } & \multicolumn{4}{|c|}{$\mathrm{m}(\%)$} \\
\hline & $0-5 \mathrm{~cm}$ & $5-10 \mathrm{~cm}$ & $10-20 \mathrm{~cm}$ & Média \\
\hline Floresta Secundária & $2,82 \mathrm{Aa}$ & 7,04 Ba & $8,45 \mathrm{Ca}$ & 6,10 \\
\hline Pastagem & $4,50 \mathrm{Aa}$ & $8,25 \mathrm{Ba}$ & $5,55 \mathrm{Ca}$ & 6,10 \\
\hline Teca $19 \mathrm{EL}$ & $2,22 \mathrm{Ac}$ & $17,29 \mathrm{Ab}$ & $27,59 \mathrm{Aa}$ & 15,70 \\
\hline Teca 19 PC & $1,53 \mathrm{Aa}$ & $3,56 \mathrm{Ba}$ & $4,18 \mathrm{Ca}$ & 3,09 \\
\hline Teca 16 EL & $1,61 \mathrm{Aa}$ & $4,49 \mathrm{Ba}$ & $4,79 \mathrm{Ca}$ & 3,63 \\
\hline Teca 16 PC & $1,71 \mathrm{Aa}$ & $2,67 \mathrm{Ba}$ & $1,35 \mathrm{Ca}$ & 1,91 \\
\hline Teca 13 EL & $1,28 \mathrm{Ac}$ & $10,80 \mathrm{Ab}$ & $13,21 \mathrm{Ba}$ & 8,43 \\
\hline Teca 13 PC & $0,43 \mathrm{Aa}$ & $1,96 \mathrm{Ba}$ & $4,66 \mathrm{Ca}$ & 2,35 \\
\hline Teca 8 EL & $7,55 \mathrm{Ab}$ & $13,35 \mathrm{Aa}$ & $14,02 \mathrm{Ba}$ & 11,64 \\
\hline Teca 8 PC & $1,87 \mathrm{Aa}$ & $1,74 \mathrm{Ba}$ & $2,84 \mathrm{Ca}$ & 2,15 \\
\hline
\end{tabular}

Médias seguidas de mesma letra, maiúscula na coluna e minúscula na linha, não diferem entre si pelo teste Scott-Knott a 5\% de probabilidade.

Os solos sob Pastagem e sob cultivo de Teca 8 anos EL apresentaram m\% mais elevada que na Floresta Secundária, podendo indicar alterações na qualidade química do solo decorrente da retirada da vegetação nativa. Enquanto, os maiores valores de $\mathrm{m} \%$ na Floresta
Secundária em relação aos cultivos de Teca com 19 anos EL e PC, 16 anos EL e PC, 13 anos EL e PC e 8 anos PC está relacionada à acidificação natural do solo promovida pela matéria orgânica.

Porém, interpretando os valores de $\mathrm{m} \%$, verificou-se que estes se enquadraram na faixa 
de baixo $(\leq 20,0 \%)$, segundo Sousa e Lobato (2004), e, portanto, indicando que o teor de $\mathrm{Al}^{3+}$ no solo pode não estar influenciando de modo significativo na acidez do solo.

Houve diferença entre os teores de Carbono Orgânico Total (COT) em todos os sistemas estudados (Tabela 12), em geral, diminuindo conforme o aumento da profundidade. Exceto no tratamento Teca $9 \mathrm{PC}$, em que os teores aumentaram de $0-5$ para $5-10 \mathrm{~cm}$, voltando a reduzir na camada de $10-20 \mathrm{~cm}$. Essa característica também foi observada por Campos et al. (2013), estudando o estoque de C em plantio convencional com três anos, plantio direto com três, com cinco e com nove anos e; Denardin et al. (2014), comparando o estoque de C em Floresta Natural, com cultivo de Eucalyptus saligna e com cultivo de Ilex paraguariensis.

Tabela 12 - COT em função dos tratamentos e das profundidades de amostragem.

Table 12 - COT in function of treatments and sampling depths.

\begin{tabular}{ccccc}
\hline \multirow{2}{*}{ Tratamento } & \multicolumn{4}{c}{ COT $\mathbf{~ ( g ~ k g} \mathbf{~}^{-1} \mathbf{~}$} \\
\cline { 2 - 5 } & $0-5 \mathrm{~cm}$ & $5-10 \mathrm{~cm}$ & $10-20 \mathrm{~cm}$ & Média \\
\hline Floresta Secundária & $5,97 \mathrm{Aa}$ & $4,44 \mathrm{Cb}$ & $3,33 \mathrm{Bc}$ & 4,58 \\
Pastagem & $6,54 \mathrm{Aa}$ & $5,78 \mathrm{Aa}$ & $4,60 \mathrm{Ab}$ & 5,64 \\
Teca 19 EL & $5,47 \mathrm{Aa}$ & $3,98 \mathrm{Cb}$ & $3,42 \mathrm{Bb}$ & 4,29 \\
Teca 19 PC & $5,57 \mathrm{Aa}$ & $4,95 \mathrm{Bb}$ & $4,44 \mathrm{Ab}$ & 4,99 \\
Teca 16 EL & $4,68 \mathrm{Ba}$ & $3,97 \mathrm{Cb}$ & $3,67 \mathrm{Bb}$ & 4,11 \\
Teca 16 PC & $5,53 \mathrm{Aa}$ & $5,41 \mathrm{Aa}$ & $4,48 \mathrm{Ab}$ & 5,14 \\
Teca 13 EL & $5,51 \mathrm{Aa}$ & $4,19 \mathrm{Cb}$ & $4,01 \mathrm{Bb}$ & 4,57 \\
Teca 13 PC & $6,21 \mathrm{Aa}$ & $4,79 \mathrm{Bb}$ & $4,04 \mathrm{Bb}$ & 5,01 \\
Teca 8 EL & $4,71 \mathrm{Ba}$ & $3,60 \mathrm{Cb}$ & $3,30 \mathrm{Bb}$ & 3,87 \\
Teca 8 PC & $5,81 \mathrm{Aa}$ & $6,05 \mathrm{Aa}$ & $5,04 \mathrm{Ab}$ & 5,63 \\
\hline
\end{tabular}

Médias seguidas de mesma letra, maiúscula na coluna e minúscula na linha, não diferem entre si pelo teste Scott-Knott a 5\% de probabilidade.

Os maiores teores de COT ocorreram na camada de $0-5 \mathrm{~cm}$ para todos os tratamentos. Com os menores valores em Teca 16 EL e Teca 8 EL.

Na camada de $5-10 \mathrm{~cm}$, os maiores valores foram observados nos tratamentos Pastagem, Teca 16 PC e Teca 8 PC e; em Pastagem, Teca 19 PC, Teca 16 PC e Teca 8 PC na camada de 10 - $20 \mathrm{~cm}$. Em geral, o sistema Pastagem apresentou os maiores teores de COT $\left(6,54 \mathrm{~g} \mathrm{~kg}^{-}\right.$ ${ }^{1}$ ), provavelmente devido ao acúmulo de esterco bovino, bem como da maior concentração de raízes, porém, sem diferença estatística para a Floresta Secundária.

$\mathrm{O}$ aumento dos teores de COT, no sistema Teca, pode ser explicado pela presença de serapilheira de baixa taxa de decomposição, já que pode ser mais lignificada e, dessa forma, demora mais para se decompor, diferentemente do sistema de floresta que possui diversidade de material a ser decomposto.
Segundo Denardin et al. (2014), esses resultados demonstram a importância da estabilidade do solo nos sistemas florestais e do aporte de resíduos vegetais, pois o manejo do solo e a ação de fatores abióticos influenciam na degradação da M.O e, assim, na concentração de C.

Sá et al. (2003) concluíram que, em relação às propriedades químicas, os teores de Carbono Orgânico (CO) e a CTC nos horizontes superficiais, representam índices de degradação de solo que podem ser adotados para estimar os efeitos ambientais de sistemas de produção agrícola em solos de tabuleiro. Portanto, ao serem utilizados para o presente trabalho podem demonstrar que, em relação à Floresta Secundária, os outros sistemas explicitam as alterações no solo após a mudança no seu uso. 


\section{Conclusões}

As alterações da cobertura vegetal nativa em sistemas de cultivo promoveram mudanças nas propriedades químicas do solo.

A camada de 0 - $5 \mathrm{~cm}$ pode ser considerada a mais fértil dentre as coberturas vegetais estudadas, pois apresentou os maiores valores de $\mathrm{pH}, \mathrm{Al}^{3+}, \mathrm{Ca}^{2+}, \mathrm{Mg}^{2+}, \mathrm{K}^{+}, \mathrm{P}$, de soma de bases, de capacidade de troca de cátions, de saturação por bases e de carbono orgânico total.

O solo sob Floresta Secundária apresentou os maiores teores de $\mathrm{Ca}^{2+}, \mathrm{K}^{+}$e de $\mathrm{P}$, os maiores valores de soma de bases, de capacidade de troca de cátions e de saturação por bases na camada de 0 - $5 \mathrm{~cm}$, em relação aos cultivos de Teca com 8 , 13, 16 e 19 anos e a Pastagem.

Os solos sob Pastagem e sob cultivo de Teca com 13 anos apresentaram os maiores teores de COT na camada de $0-5 \mathrm{~cm}$, em razão da maior quantidade de raízes da Brachiaria brizantha nessa camada da área de Pastagem e da serapilheira com baixa taxa de decomposição formada pelo cultivo de Teca.

\section{Referências Bibliográficas}

BRASNORTE MT. Geografia. 2016. Disponível em: $<$ http://www.brasnorte.mt.gov.br/ConhecaNossa-Cidade/Geografia/ >. Acesso em: $21 \mathrm{de}$ dezembro de 2016.

CAMPOS, L. P. et al. Estoque e frações de carbono orgânico em Latossolo Amarelo submetido a diferentes sistemas de manejo. Pesquisa Agropecuária Brasileira, v. 48, n. 3, p. 304-312, 2013.

CARDOSO, E. L. et al. Qualidade química e física do solo sob vegetação arbórea nativa e pastagens no Pantanal Sul-mato-grossense. Revista Brasileira de Ciência do Solo, v. 35, n. 2, p. 613-622, 2011.

CARNEIRO, M. A. C. et al. Atributos físicos, químicos e biológicos de solo de cerrado sob diferentes sistemas de uso e manejo. Revista Brasileira de Ciência do Solo, v. 33, p. 147157, 2009.

DENARDIN, R. B. N. et al. Estoque de carbono no solo sob diferentes formações florestais, Chapecó - SC. Ciência Florestal, v. 24, n. 1, p. 59-69, 2014.

EMBRAPA. Centro Nacional de Pesquisa de Solos. Manual de Métodos de Análise de Solo. 2. ed. Rio de Janeiro: EMBRAPA SOLOS, 1997. $212 \mathrm{p}$.

EMBRAPA. Centro Nacional de Pesquisa de Solos. Sistema Brasileiro de Classificação de Solos. 2. ed. Rio de Janeiro: EMBRAPA SOLOS, 2006. 306 p.

FERREIRA, D. F. Sisvar: versão 4.6. Lavras: UFLA, 2003.

LIMA, S. S. et al. Atributos químicos e estoques de carbono e nitrogênio em Argissolo vermelhoamarelo sob sistemas agroflorestais e agricultura de corte e queima no norte do Piauí. Revista Árvore, v. 35, n. 1, p. 51-60, 2011.

LOPES, E. L. N. et al. Características químicas de um Gleissolo sob diferentes sistemas de uso, nas margens do rio Guamá, Belém, Pará. Boletim do Museu Paraense Emilio Goeldi. Ciências Naturais, v. 1, n. 1, p. 127-137, 2006.

MELLONI, R. et al. Avaliação da qualidade de solos sob diferentes coberturas florestais e de pastagem no sul de Minas Gerais. Revista Brasileira de Ciência do Solo, v. 32, p. 24612470, 2008.

RAMOS, B. Z. et al. Doses de gesso em cafeeiro: influência nos teores de cálcio, magnésio, potássio e pH na solução de um Latossolo Vermelho distrófico. Revista Brasileira de Ciência do Solo, v. 37, n. 4, p. 1018-1026, 2013.

SÁ, R. C.; PEREIRA, M. G.; FONTANA, A. Características físicas e químicas de solos de 
tabuleiros em Sooretama (ES). Floresta e Ambiente, v. 10, n. 3, p. 95-99, 2003.

SALGADO, B. G. et al. Avaliação da fertilidade dos solos de sistemas agroflorestais com cafeeiro em lavras-MG. Revista Árvore, v. 30, n. 3, p. 343-349. 2006.

SANTOS, A. C.; SALCEDO, I. H. Relevo e fertilidade do solo em diferentes estratos da cobertura vegetal na bacia hidrográfica da represa vaca brava, Areia-PB. Revista Árvore. v. 34, n. 2, p. 277-285. 2010.

SILVA, L. A. G. C. Biomas presentes no estado do Tocantins. Tocantins: Biblioteca Digital da Câmara dos Deputados, 2007. 10p.

SILVA, R. C. et al. Alterações nas propriedades químicas e físicas de um chernossolo com diferentes coberturas vegetais. Revista Brasileira de Ciência do Solo, v. 31, n. 1, p. 101-107, 2007.

SILVA JUNIOR, C. A.; BOECHAT, C. L.; CARVALHO, L. A. Atributos químicos do solo sob conversão de floresta Amazônica para diferentes sistemas na região Norte do Pará, Brasil. Bioscience Journal, v. 28, n. 4, p. 566572, 2012.

SOUSA, D. M. G.; LOBATO, E. Cerrado: correção do solo e adubação. Planaltina: EMBRAPA CERRADOS, 2004. 416 p.

YEOMANS, A.; BREMNER, J. M. A rapid and precise method for routine determination of organic carbon in soil. Communication in Soil Science and Plant Analysis. v. 19. p. 14671476. 1988. 